from hospital and we followed the first admission of each. 28533 (36\%) patients were readmitted within 1-year and 8439 (11\%) within 30 days. Patients readmitted within 30 days were more likely to be male (53\% vs $50 \%$; $<<0.001$ ), older (72.7 vs 71.6 yrs; $\mathrm{p}<0.001$ ), live in a more deprived area (55\% vs $51 \%$ highest quintile; $p<0.001$ ) and much more likely to die (30-day mortality $11 \%$ vs $3 \%$; $<<0.001$; and 1 -year mortality $40 \%$ vs $20 \%$; $<0.001$ ). For the index admission, patients readmitted within 30 days were more likely to have been admitted via A\&E rather than via their GP (74\% vs $70 \%$; $\mathrm{p}<0.001)$, more likely to have had a LRTI ( $88 \%$ vs $83 \%$; $<<0.001)$, had a longer median length of stay ( 6 vs 5 days; $p<0.001$ ) and were more likely to have been discharged from the care of a chest physician (44\% vs $40 \%$; $p<0.001$ ). Hospitals with the lowest inpatient mortality during the first admission had the highest 30-day readmission rate $(r=0.22 ; p<0.01)$. Early readmission would appear to be driven by demographics, severity of the index admission and disease severity which cannot be influenced by care delivery or organisation of care. It's possible that better care-keeping more patients alive during their index admission-may lead to those hospitals being penalised for a higher 30-day readmission rate.

\section{P100 DESIGNING AND IMPLEMENTING A COPD DISCHARGE CARE BUNDLE}

\section{doi:10.1136/thoraxjnl-2011-201054c.100}

${ }^{1} \mathrm{~N}$ S Hopkinson, ${ }^{2} \mathrm{C}$ Englebretsen, ${ }^{1} \mathrm{~N}$ Cooley, ${ }^{3} \mathrm{~K}$ Kennie, ${ }^{1} \mathrm{M}$ Lim, ${ }^{2} \mathrm{~T}$ Woodcock, ${ }^{2} \mathrm{~A}$ Laverty, ${ }^{2} \mathrm{~S}$ Wilson, ${ }^{4} \mathrm{~S}$ L Elkin, ${ }^{2} \mathrm{C}$ Caneja, ${ }^{3} \mathrm{C}$ Falzon, ${ }^{2} \mathrm{H}$ Burgess, ${ }^{2} \mathrm{D}$ Bell, ${ }^{2} \mathrm{D}$ Lai. ${ }^{1}$ NIHR Respiratory Biomedical Research Unit of Royal Brompton and Harefield NHS Trust and Imperial College, London, UK; ${ }^{2}$ North West London Collaboration for Leadership in Applied Health Research and Care, Chelsea and Westminster NHS Foundation Trust, London, UK; ${ }^{3}$ Central London Community Healthcare, London, UK; ${ }^{4}$ Imperial Healthcare NHS Trust, London, UK

Problem Acute exacerbations of COPD are a major cause of hospital admission and readmission. National surveys have revealed significant differences in patient outcomes which are likely to be due to variations in care.

Design A care bundle, comprising a short list of evidence-based practices to be implemented prior to discharge for all patients admitted with this condition was developed, based on a review of National Guidelines and other relevant literature, expert opinion and patient consultation. Implementation was then piloted using action research methodologies in an urban acute hospital.

Key measures for improvement Improvements in process measures based around the items in the care bundle were the provision of smoking cessation advice, referral for pulmonary rehabilitation, and review of inhaler technique. A $72 \mathrm{~h}$ post-discharge phone call was evaluated. Data from the first year of the bundle were compared with the year prior to its initiation including 30-day readmission rate.

Strategies for change The care bundle was launched with events to ensure staff awareness. Weekly meetings were held with review of bundle compliance so that areas for attention could be addressed promptly. A plan, do, study, act (PDSA) cycle approach was used. Examples included ongoing support to develop ward nurses' knowledge of correct inhaler use, nurses attending pulmonary rehabilitation sessions and the use of a "safe discharge" checklist.

Effects of change Referral to pulmonary rehabilitation increased by $158 \%$ and compliance with assessment for smoking cessation was $100 \%$. Nurse confidence in inhaler technique improved. Roughly $10 \%$ of post discharge phone calls identified a cause for concern. The 30 -day readmission rate was $10.8 \%$ for patients where the bundle was used $(n=94)$ compared to $16.4 \%$ where it was not $(n=365)$ (95\% CI for difference $-2.1 \%$ to $13.2 \%$ ). The bundle has been accepted onto the list of CQUIN measures by London SHA and is being rolled out to further sites.
Lessons learnt Actively involving all staff is vital to ensure that the changes introduced are understood and the process followed. Implementation of a care bundle has the potential to produce a dramatic improvement in compliance with optimum health care measures.

\section{P101 A SURVEY OF EMERGENCY OXYGEN GUIDELINE IMPLEMENTATION AMONG ALL 15 UK AMBULANCE SERVICES IN EARLY 2011}

doi:10.1136/thoraxjnl-2011-201054c.101

${ }^{1} \mathrm{~F}$ P Moore, ${ }^{2} \mathrm{~A}$ G Davison, ${ }^{1} \mathrm{~J}$ Smith, ${ }^{3} \mathrm{~B}$ R O'Driscoll. ' $L o n d o n$ Ambulance Service, London, UK; ${ }^{2}$ Southend University Hospital, Southend, UK; ${ }^{3}$ Salford Royal University Hospital, Salford, UK

The British Thoracic Society (BTS), together with 21 other societies published a UK guideline for emergency oxygen use in 2008. This guideline was endorsed by the Joint Royal Colleges Ambulance Liaison Committee (JRCALC) who produced new oxygen guidance for ambulance crews in April 2009. We have conducted a survey of implementation of this guidance among UK Ambulance Services in early 2011. A questionnaire was sent to the Medical Directors of all 15 UK Ambulance Services and all, or their nominated deputies responded. Eleven of fifteen respondents reported full implementation of the 2009 JRCALC oxygen guidance throughout their service and ten of these reported that all relevant staff are trained in this area of practice. However, an informal survey of about 100 front line ambulance crews in one of these areas found that none were aware of the 2009 JRCALC document so the above figures may be aspirational and not yet achieved at operational level. Four services reported that they have completed audits of guideline implementation and a further three services are planning audits. All 15 services reported that $81 \%-100 \%$ of response vehicles were equipped with oximeters and the availability of oxygen masks was as follows: 15/15 reservoir masks; 12/15 simple face masks, 12/15 $28 \%$ Venturi masks, $6 / 15$ 24\% Venturi masks, 10/15 nasal cannulae. However, the informal survey of front-line staff from one ambulance service showed that Venturi masks were not actually available at operational level although the Regional response indicated universal availability. A separate survey found that no UK ambulance service has access to air cylinders, compressors or ultrasonic nebulisers for COPD patients so all nebulised treatment is oxygendriven. Six of 15 services reported that they had protocols to limit the duration of oxygen-driven nebuliser therapy for COPD patients. Two services have a record of all patients in their area who have an Oxygen Alert card. This survey suggests that UK Ambulance Services are taking steps to implement the BTS and JRCALC emergency oxygen guidance but it is unclear how much advice and equipment had been cascaded to front-line staff by Spring 2011.

\section{P102 A SURVEY OF HOME OXYGEN PROVISION ACROSS LONDON}

doi:10.1136/thoraxinl-2011-201054c.102

A C Davidson, S Williams, N Baxter, E Morris, L Restrick. London Respiratory Team, NHS London, Victoria, London, UK

Introduction In preparing for 2012, we undertook a survey of current oxygen provision across London.

Methods A questionnaire was sent electronically (Survey Monkey) to 100 BTS Oxygen champions, oxygen leads in primary and secondary care, PCT prescribing advisors and community respiratory nurses around London.

Results From 60 returns the main findings were:

1. $75 \%$ of LTOT prescriptions initiated by hospitals yet no follow-up in $35 \% .70 \%$ community teams reported "not at all" and "only sometimes" being informed about new starters 
by GPs and hospitals. $<50 \%$ took action when concordance reports showed variance (under or over use) from prescribed treatment.

2. Lack of ability to detect hypoxic patients with $>20 \%$ community healthcare sites not having access to an oximeter.

3. Fire safety officers are rarely advised about the persistent smoker (only 16\%) despite the potential risk to patients, their families and the general public. Local guidance on appropriate steps to take is rare (35\%). 3 HOS units denied LTOT for smokers and one assessed this by exhaled carbon monoxide measurement.

4. A variety of methods for protecting patients from excessive oxygen are favoured but use appears limited. When asked what policy respondents favoured, universal precaution (as promoted by ambulance guidelines) was most popular (60\%) while $20 \%$ favoured oxygen cards and $20 \%$ patient specific protocols (PSP).

5. A specific local policy for removing oxygen when no longer indicated or used is rare $(<25 \%)$. This, coupled with inadequate follow-up of patients started on oxygen during hospital admission, suggests significant waste with the current oxygen provision.

6. Respondents indicated guidance on oxygen removal, contract monitoring, assessment for ambulatory oxygen and training in arterial or capillary blood gases as being required.

Conclusions Problems in healthcare coordination, public and patient safety and in removing oxygen once ordered were common. There is a need to integrate hospital and community teams and to prepare for safe mobilisation and contract management so that a quality home oxygen service can be provided in the future.

\section{P103 THE PRACTICE OF PRESCRIPTION OF LONG-TERM OXYGEN THERAPY TO PATIENTS WHO CONTINUE TO SMOKE}

doi:10.1136/thoraxjnl-2011-201054c.103

C K Y Lee, J Noble, D A Wales, R M Jones. Nevill Hall Hospital, Abergavenny, UK

Introduction Although patients who receive long-term oxygen therapy (LTOT) are discouraged from smoking, a substantial proportion of active smokers receive this therapy. There is limited published guidance addressing this issue.

Methods The authors designed a questionnaire consisting of six multiple choice type questions based on the practice of the prescription of LTOT, particularly focusing on the provision of LTOT to active smokers. This was sent to all consultant respiratory physicians in Wales.

Results 35 out of 45 (73\%) questionnaires were returned. 17 physicians (49\%) did not prescribe LTOT to active smokers, whereas $18(51 \%)$ did. For those who did not prescribe LTOT to smokers, this was according to departmental policy in 9 (53\%) and personal practice in $8(47 \%)$. In this group, all respondents stated the reason for not prescribing oxygen was due to risk of harm to the individual, with the majority (88\%) also citing risk to other household members. Other reasons included the reduced likelihood of benefit and effectiveness in smokers (35\%) and as an incentive to smoking cessation. 7 (41\%) of this group would consider prescribing oxygen to smokers as palliation for severe hypoxia and malignancy. Of the physicians who prescribed LTOT to active smokers, 16 (89\%) felt there was existing evidence suggesting benefit in both smokers and non-smokers. $10(55 \%)$ of this group considered it unethical to deny patients LTOT and 11 (61\%) felt it was the responsibility of individual patients. LTOT was prescribed only after risk and capacity assessment. Contraindications to prescribing LTOT in this group included cognitive impairment and inability to comprehend the risks. $22(63 \%)$ regularly monitored patients' smoking status while on LTOT. Of these, the majority (68\%) did so by history alone. 6 (27\%) performed Carbon Monoxide monitoring. 31 respondents $(89 \%)$ routinely counselled patients on risks of fire and burns with continued smoking on LTOT. 22 respondents (63\%) had seen burns or injuries from smoking with LTOT on at least one occasion.

Conclusion There are substantial variations in practice among respiratory physicians across Wales and likely nationwide. National guidance on this particular issue needs to be addressed to ensure standardisation of care.

\section{P104 SOUTHAMPTON CITY: FINDING THE MISSING MILLIONS AND REDUCING ADMISSIONS THROUGH MEDICAL INTELLIGENCE}

doi:10.1136/thoraxjnl-2011-201054c.104

S C Bourne, T Wikinson, H Kruk, K Austin, J Watson, N James. Southampton University Hospitals NHS Trust, Southampton, UK

Background Southampton City PCT was highlighted in a BLF report as the only hotspot on the South Coast with a population at high risk of hospital admissions with an acute exacerbation of COPD (AECOPD). The reasons stated were a low number of registered COPD patients $(1.5 \%)$, high smoking prevalence $(32 \%)$ and deprivation. Predicted levels of COPD from local healthcare modelling were $6 \%$. The trust was also experiencing a year on year increase of $15 \%$ in admissions for an acute exacerbation of COPD.

Method Southampton University Hospitals developed a primary care support team led by consultant respiratory physicians with support from respiratory nurse specialists to support primary care in the diagnosis and management of COPD. The education was delivered in a variety of formats from interactive lectures to groups of primary care doctors and nurses, small spirometry educational sessions with practice nurses, within practice education, locally led COPD diploma modules, and the development of a website (http:// www.copdeducation.org.uk). The audit was performed on all admissions coded as COPD Exacerbation from the last 3 years and all notes reviewed to confirm diagnosis.

Results During the year long project COPD prevalence (from QOF) rose from $1.5 \%$ to $2.27 \%$; an increase of $50 \%$. The audit revealed several trends (1) admissions through the ED department rose by 10\% each year from 2007 to 2010; (2) patients increasingly self presented to secondary care and (3) a large proportion of the admissions $(22 \%)$ were due to a small number of frequent attendees (34 patients responsible for 176 admissions). We cohorted the frequent attendees and with a combination of discovery interviews and nursing/clinical support reduced admissions in this cohort by $78 \%$ ). During this period COPD admissions reduced by $19 \%$ and 30 day readmission reduced from $13 \%$ to just $1.7 \%$ !

Conclusion Through the process of primary care education supported with other teaching modalities the diagnosis of COPD improved in Southampton. Medical intelligence of the admitted population combined with an appropriate intervention can reduce COPD admissions. This is just one of the ways the finding the missing millions part of the national strategy can be delivered.

Funding The Health Foundation (SHINE).

\section{P105 DIRECT ACCESS PULMONARY FUNCTION TESTING FOR PRIMARY CARE}

doi:10.1136/thoraxjnl-2011-201054c.105

${ }^{1} \mathrm{~K}$ E Backler, ${ }^{1} \mathrm{~L}$ Leech, ${ }^{1} \mathrm{M}$ Purdon, ${ }^{2} \mathrm{D}$ Roberts, ${ }^{3} \mathrm{Z}$ Lord, ${ }^{1} \mathrm{R}$ C Buttery. ${ }^{1}$ Hinchingbrooke Hospital NHS Trust, Huntingdon, UK; ${ }^{2}$ Great Staughton Surgery, Great Staughton, UK; ${ }^{3}$ National Lung Improvement Programme, London, UK

Hinchingbrooke Hospital is a Diagnostic pilot site for the NHS Lung Improvement Programme, providing direct access from local GP 